Supporting Information:

\title{
Graphene Oxide Decorated with Rh Nanospheres for Electrocatalytic Water Splitting
}

Shankar S. Narwade, Shivsharan M. Mali, Vijay S. Sapner, Bhaskar R. Sathe*

Department of Chemistry, Dr. Babasaheb Ambedkar Marathwada University, Aurangabad-431 004 (MS) India.

Email-bhaskarsathe@gmail.com

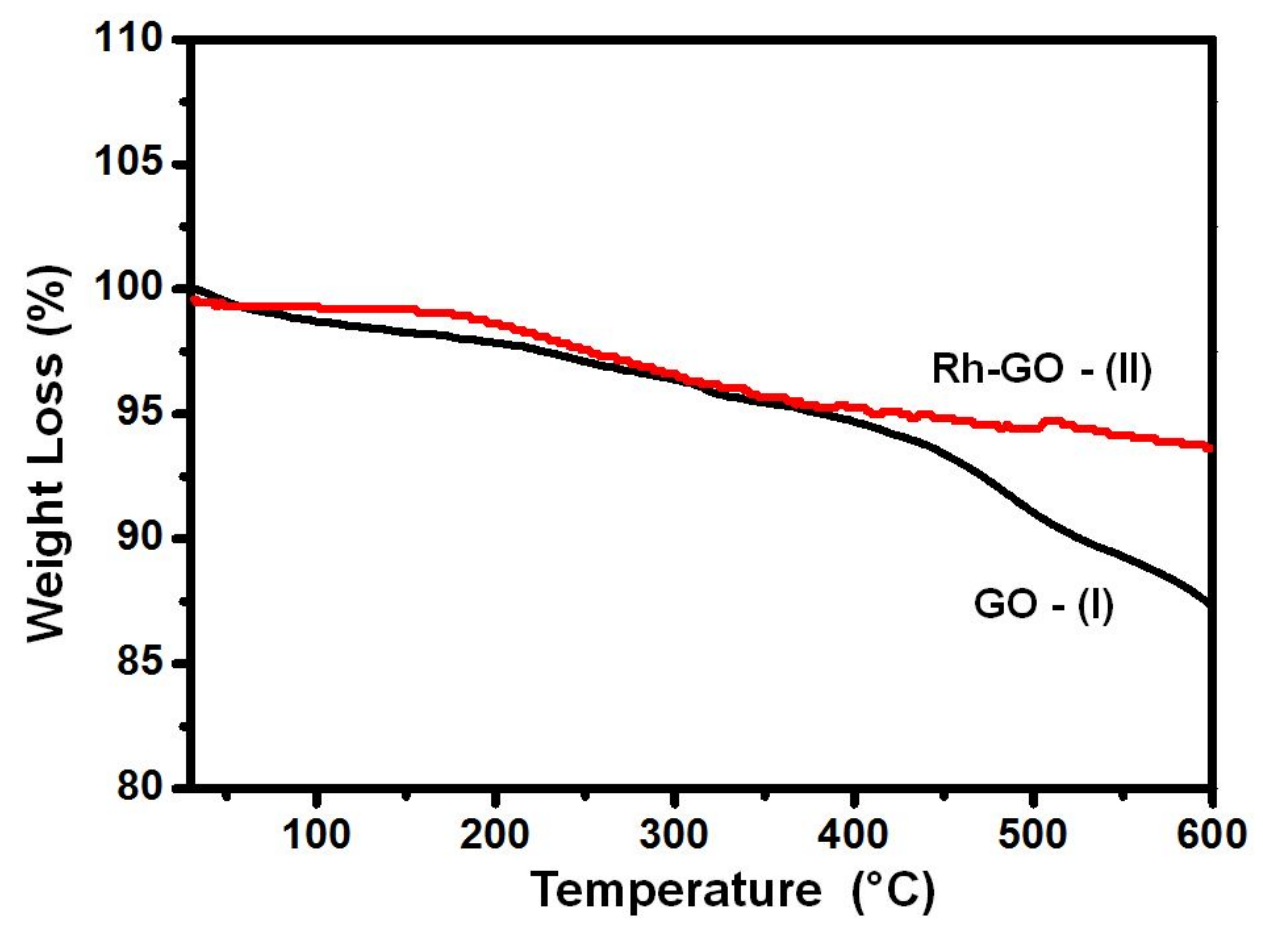

Figure S1 TGA curves annealed under air atmosphere of (I) GO, (II) Rh-GO hybrid electrocatalytic system. 


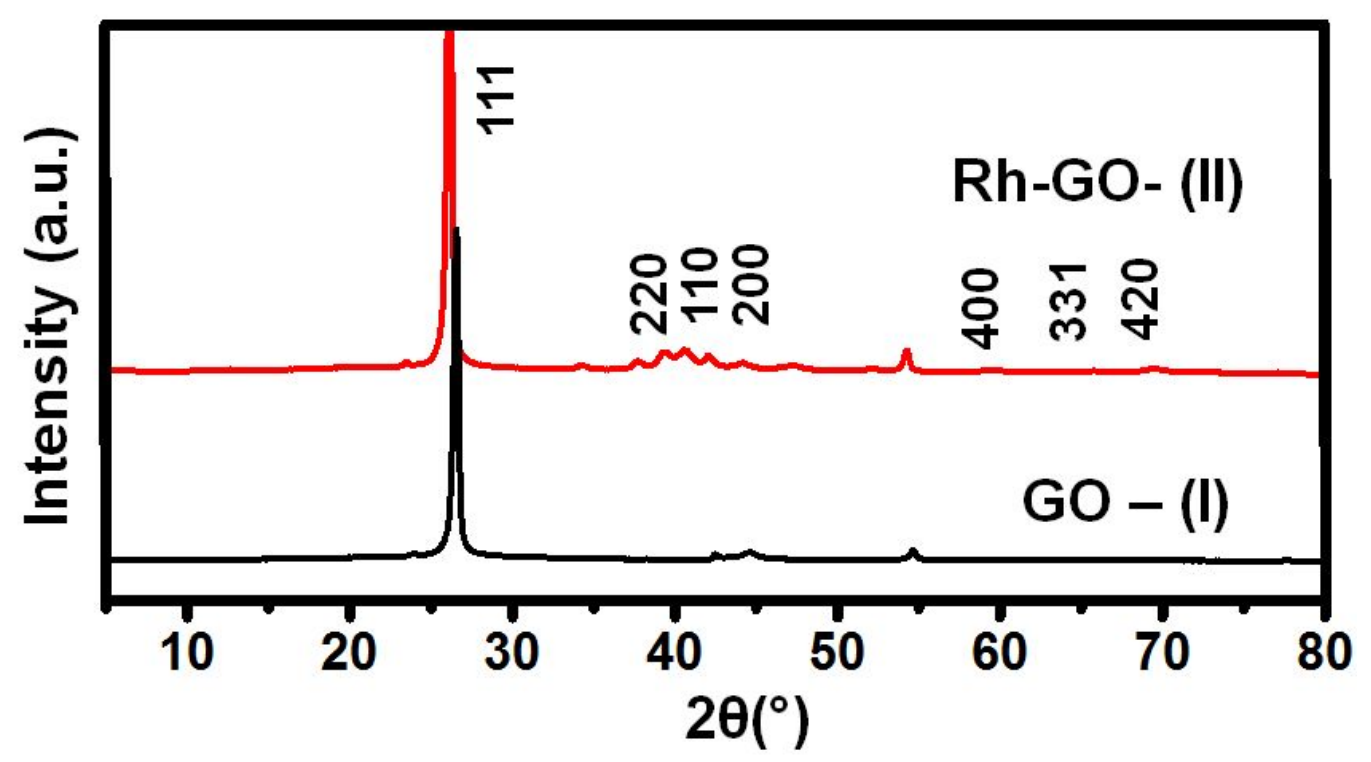

Figure S2. Superimposed XRD Patterns for GO (I) and Rh-GO hybrid nanocatalyst (II).

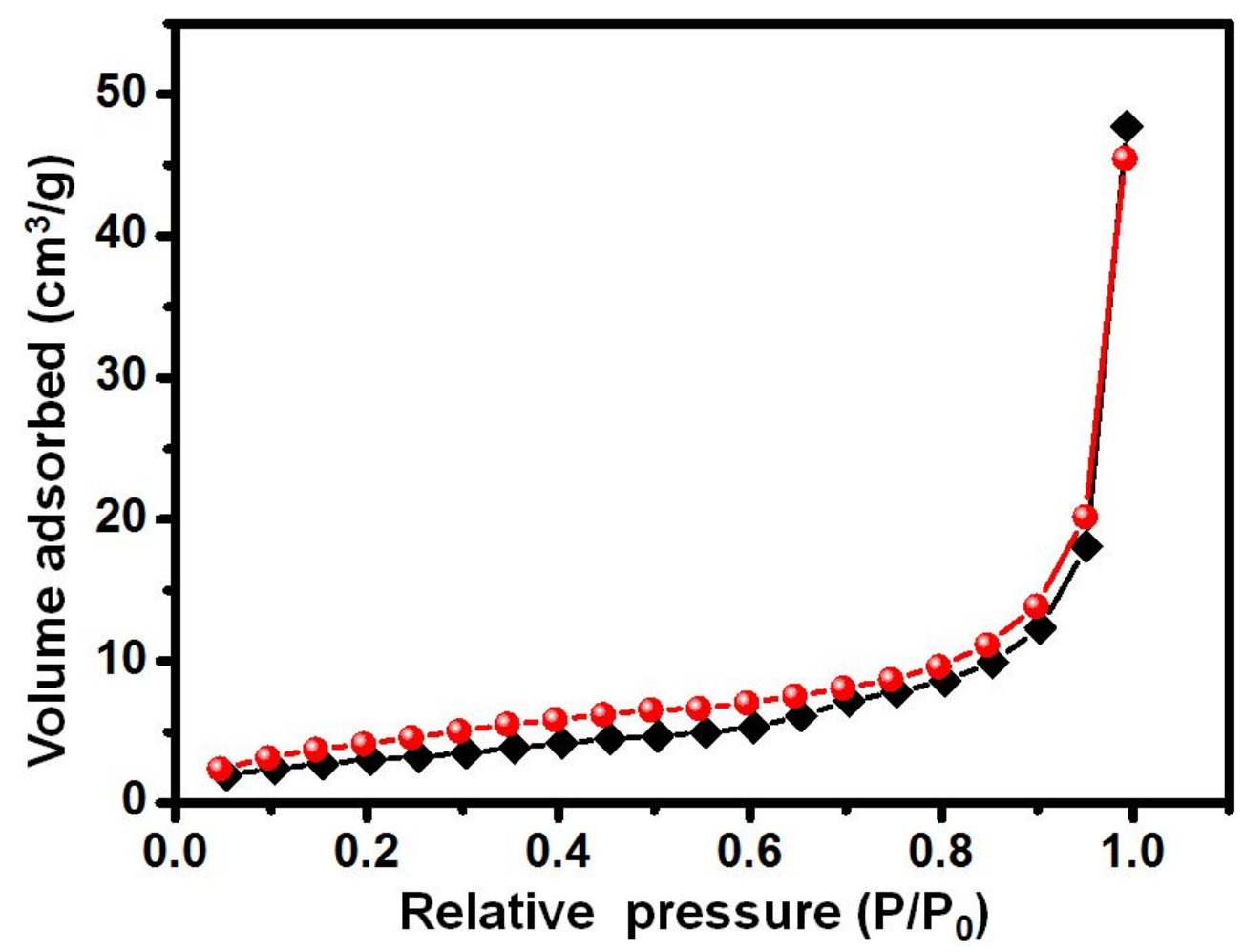

Figure S3: Nitrogen adsorption/desorption isotherms for as-synthesized Rh-GO composite nanomaterials having a pore size of $(\sim 13 \mathrm{~nm})$ Rh-doped carbon nanomaterials, whereas, the adsorption (red line) and desorption process (black line) of the electrocatalysts. 


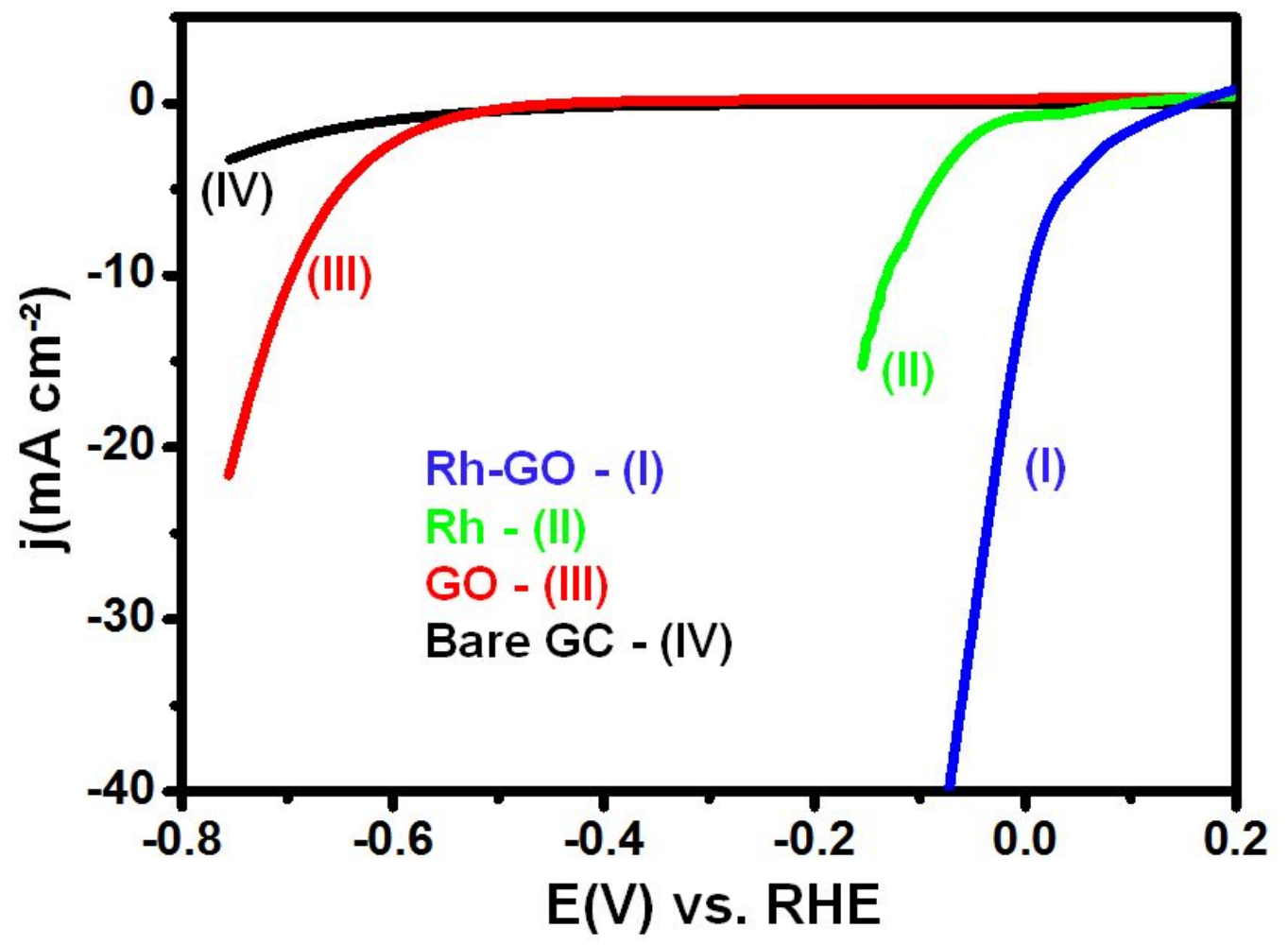

Figure S4. Superimposed cathodic polarization LSV curve for (I) Rh-GO, (II) Rh NPs, (III) functionalized GO and (IV) bare GC shows in $\mathrm{H}_{2} \mathrm{SO}_{4} 0.5 \mathrm{M}$ for HER.

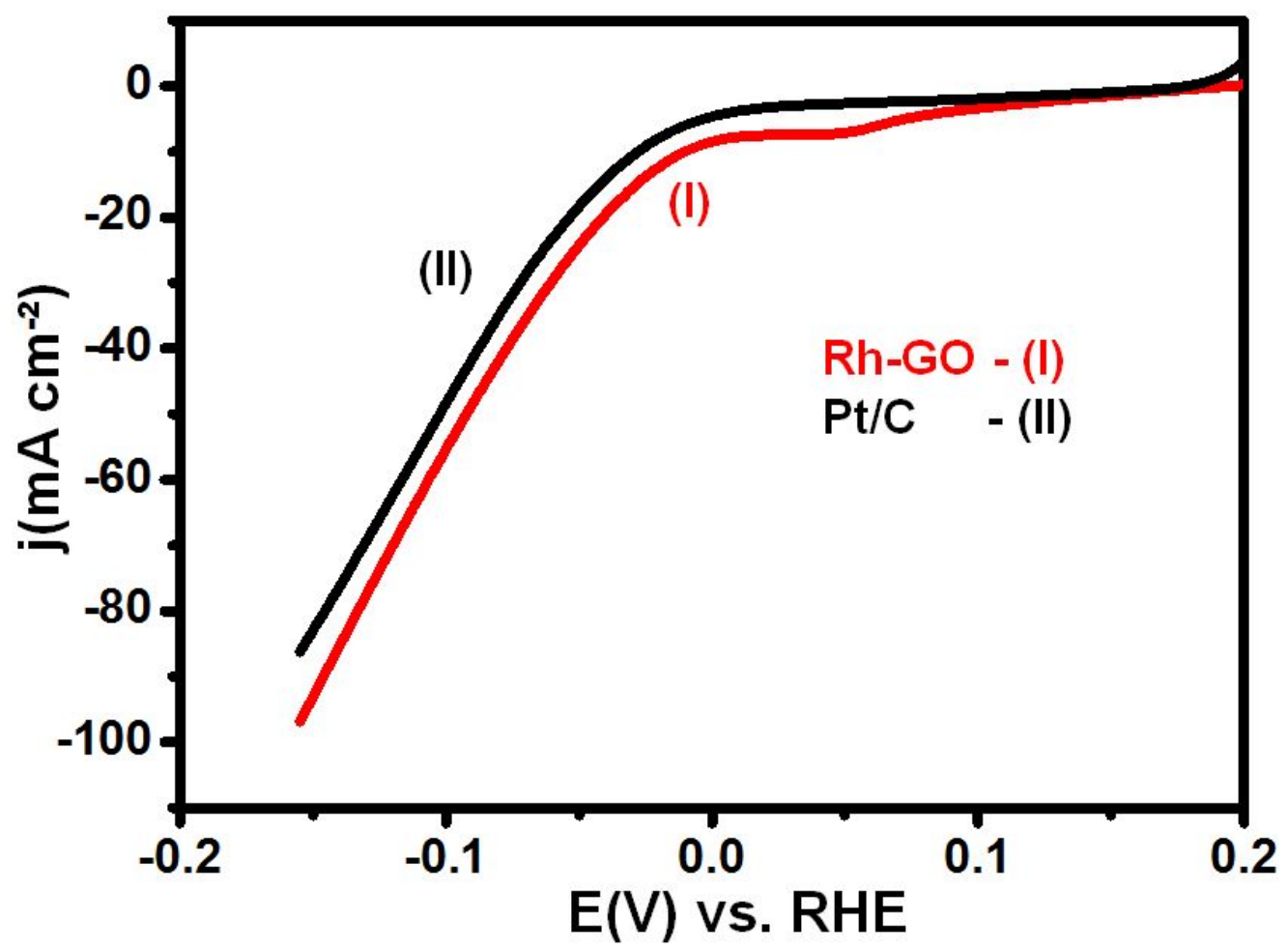

Figure S5. Linear sweep voltammograms of hydrogen evolution reaction on the Rh-GO (I) and commercial Pt/C electrode (II). Scan rate of $50 \mathrm{mV} / \mathrm{s}^{-1}$ with $0.5 \mathrm{M} \mathrm{H}_{2} \mathrm{SO}_{4}$ as the supporting electrolyte. 


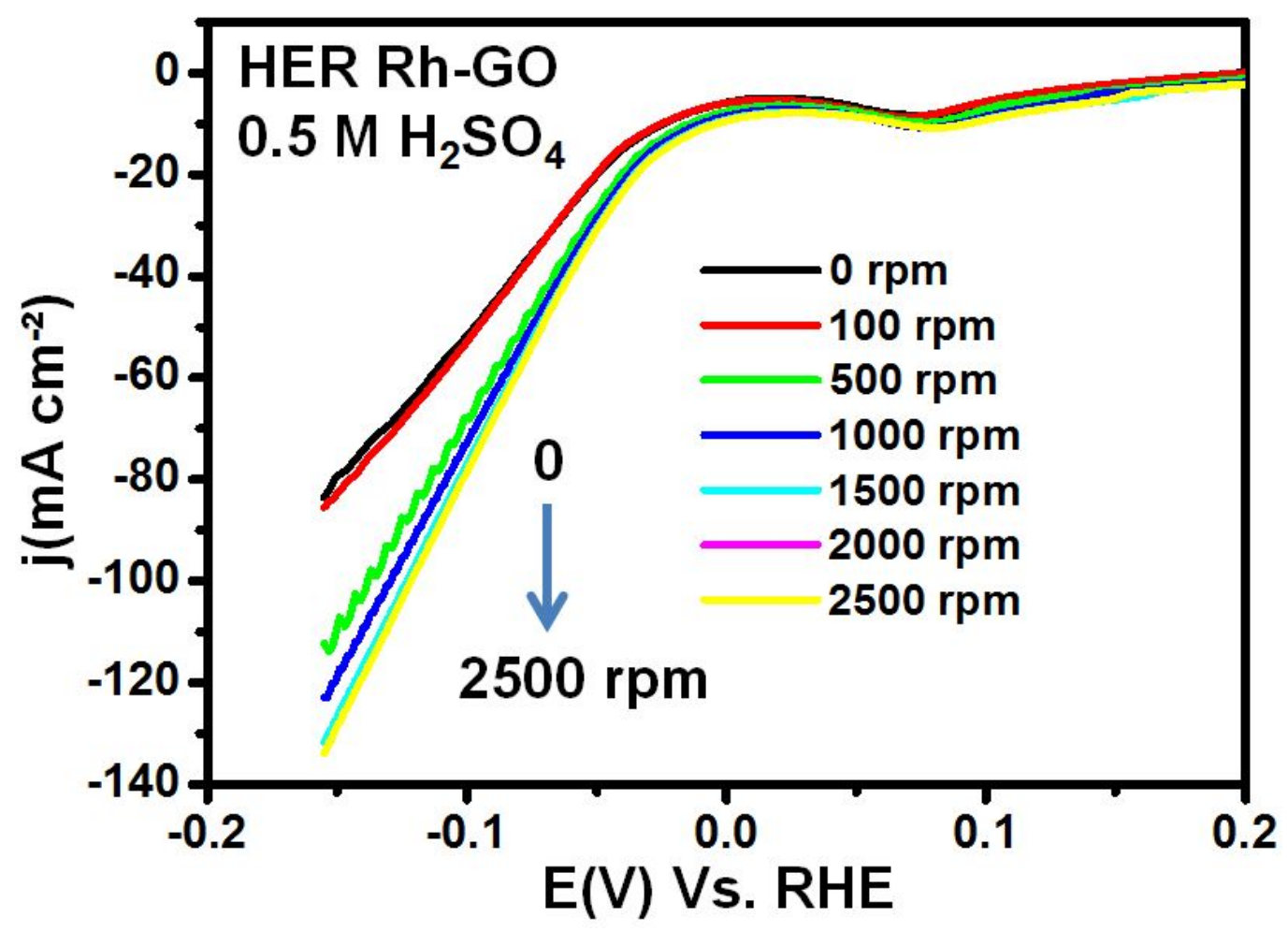

Figure S6. Superimposed linear sweep voltammograms (LSVs) for Rh-GO electrocatalyst using RRDE increases the HER activity with rotations (0 to $2500 \mathrm{rpm}$ ).

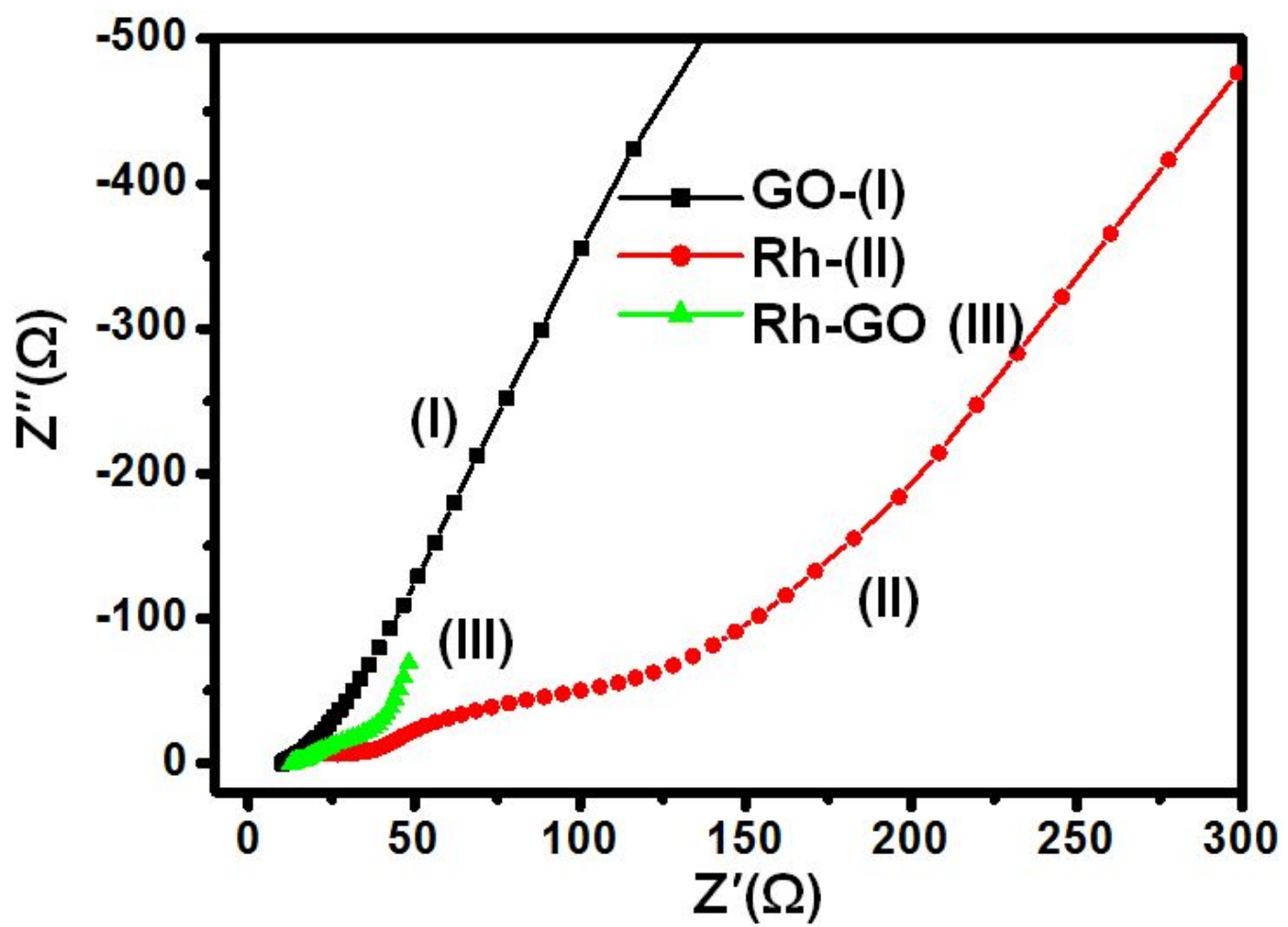

Figure S7. The Electrochemical impedance spectroscopy (EIS) curves of GO - I, Rh - II and Rh-GO III. 


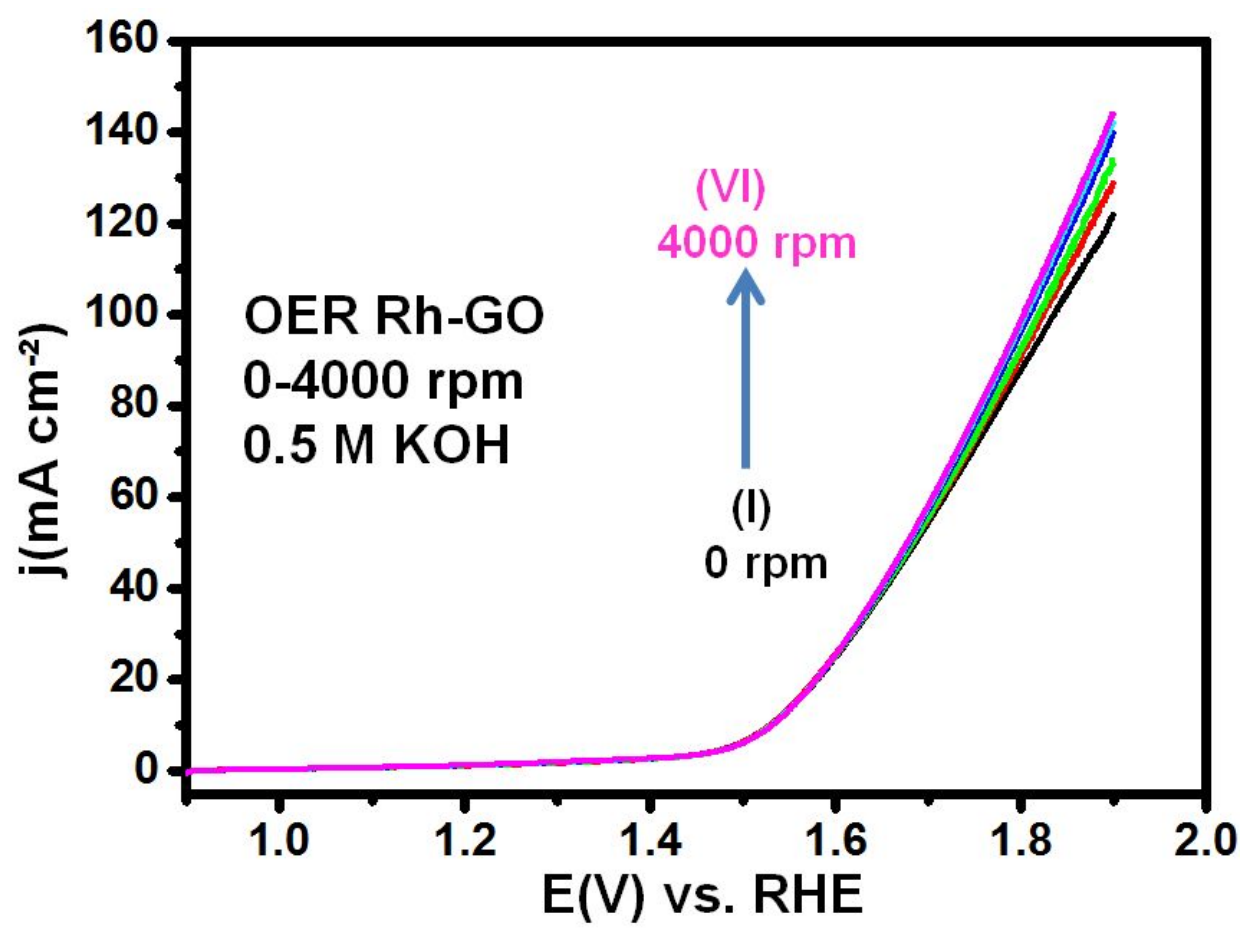

Figure S8. Superimposed linear sweep voltammograms (LSVs) for Rh-GO electrocatalyst using RRDE increases the OER activity with rotations (0 to $4000 \mathrm{rpm}$ ).

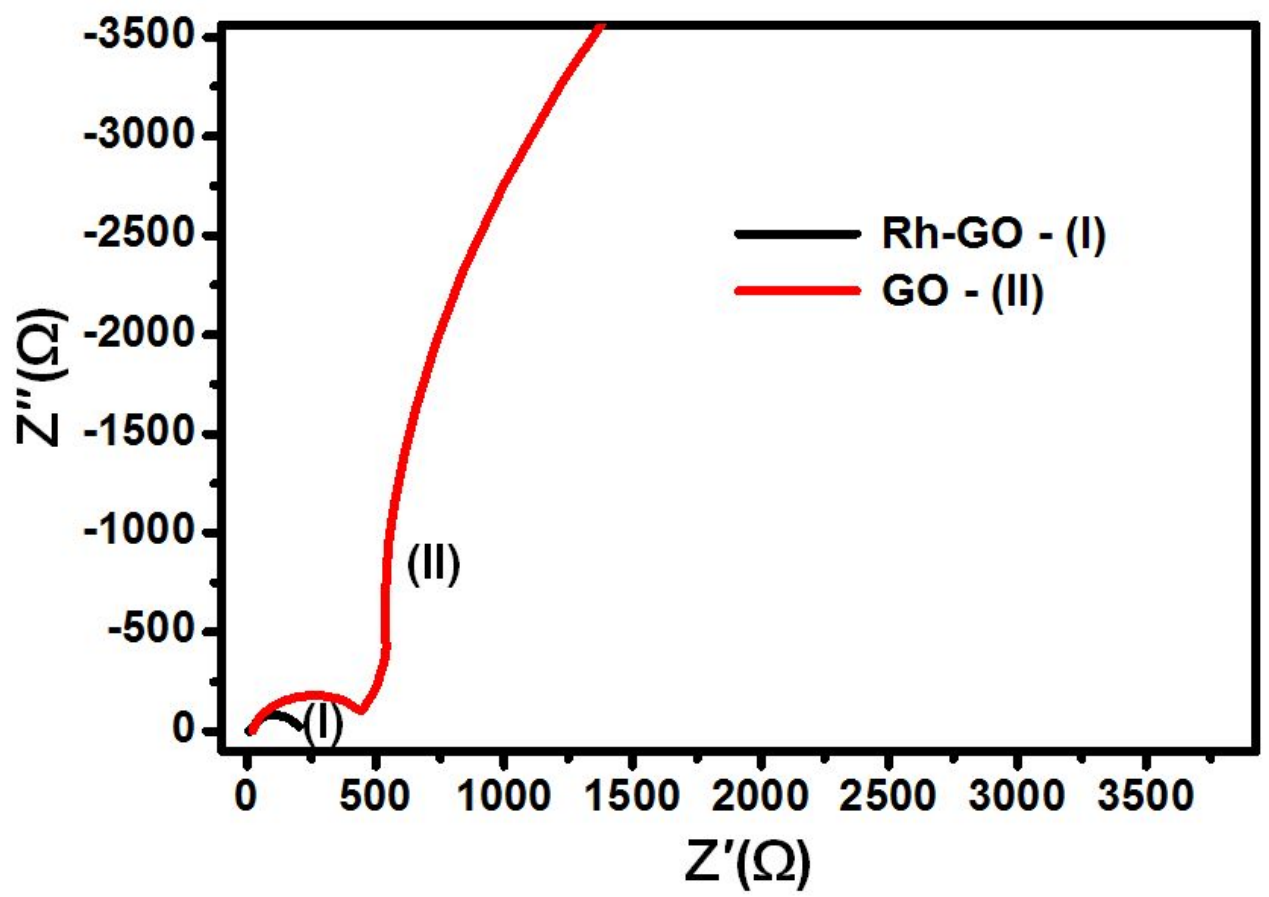

Figure S9. The Electrochemical impedance spectroscopy (EIS) curves of Rh-GO - I and GO - II. 


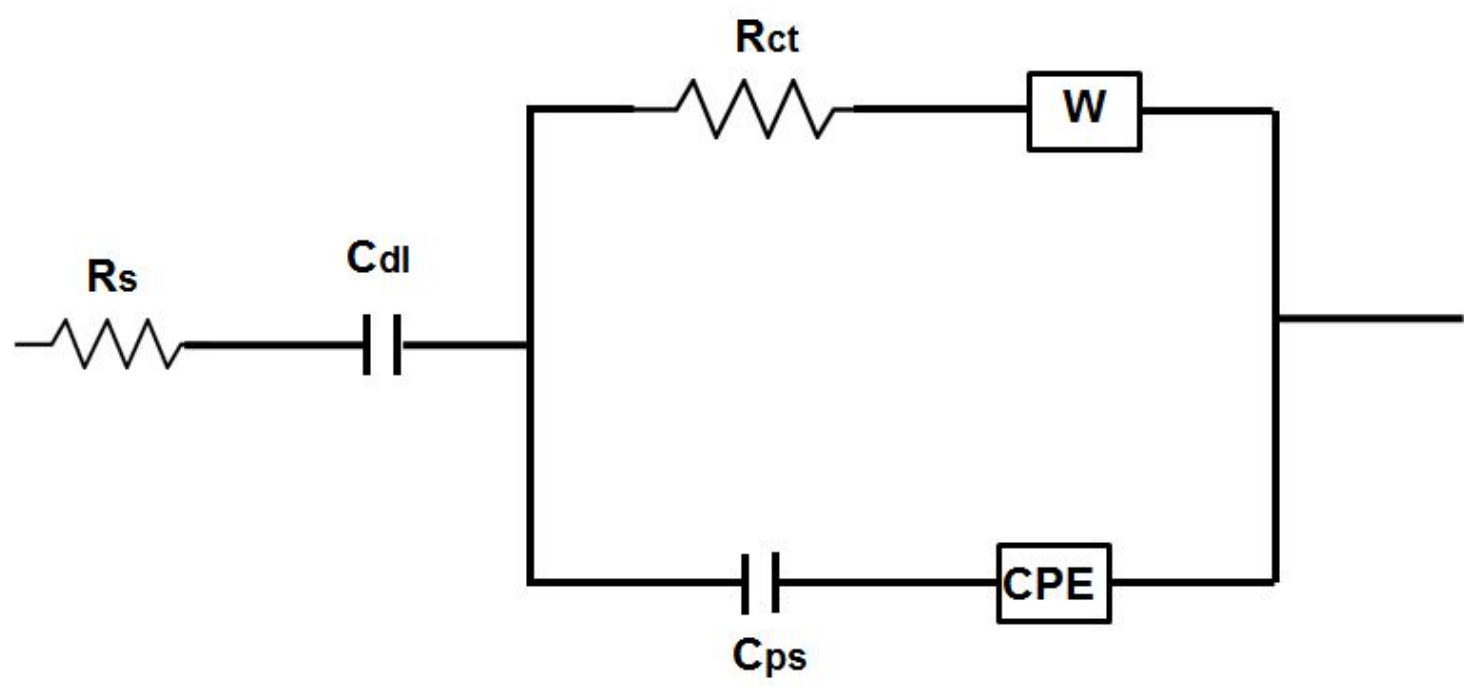

Figure S10. Equivalent circuit of Rh-GO for HER $0.5 \mathrm{M} \mathrm{H}_{2} \mathrm{SO}_{4}$ solution consisting of a solution resistance (Rs), a charge-transfer resistance (Rct) with Warburg element (W), a pseudo capacitive element (Cp), and a constant-phase element (CPE).

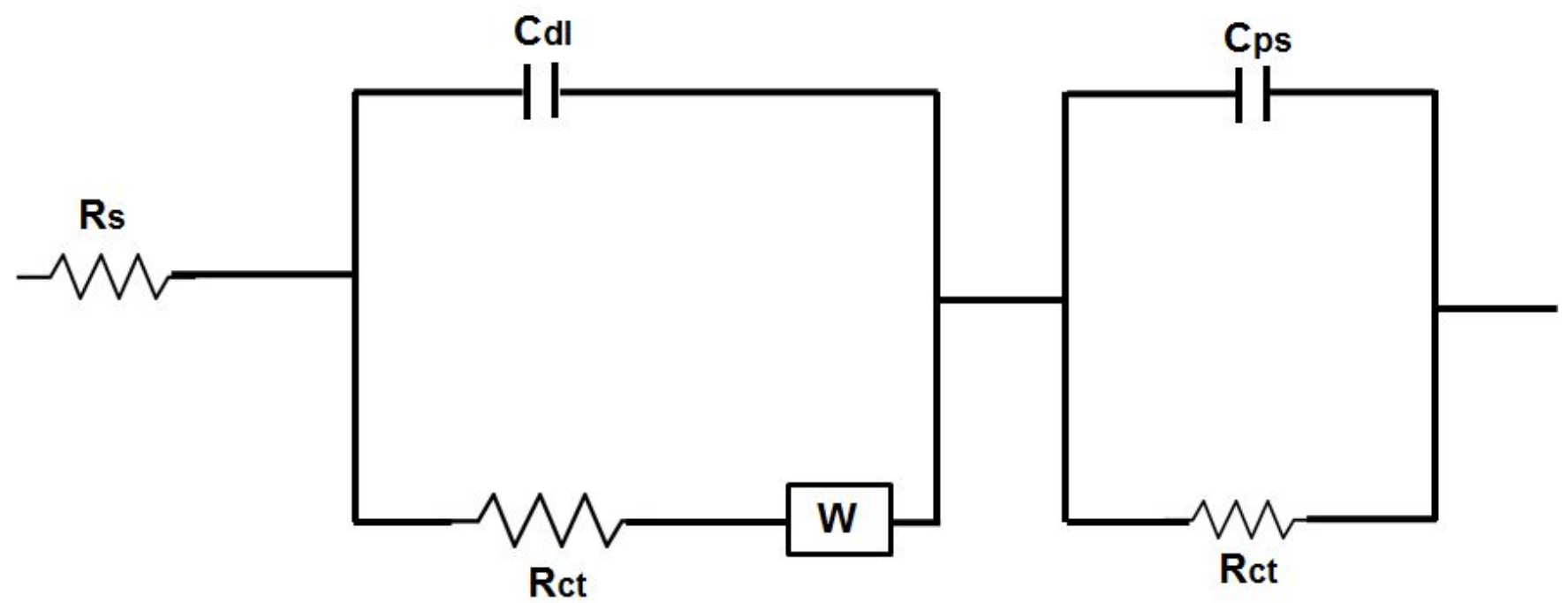

Figure S11: Equivalent circuit of Rh-GO for OER $0.5 \mathrm{M} \mathrm{KOH}$ solution consisting of a solution resistance (Rs), a charge-transfer resistance (Rct) with Warburg element (W), a pseudo capacitive element (Cp), and a constant-phase element (CPE). 
Table S1: Evaluation summary of Hydrogen Evolution Reaction belongs to state-of-the-art electrocatalysts reported in kinds of literature survey and present work.

\begin{tabular}{|c|c|c|c|c|c|}
\hline $\begin{array}{l}\text { Sr. } \\
\text { No. }\end{array}$ & Catalysts & Electrolytes & $\begin{array}{c}\text { Overpotential } \\
@ \mathbf{j}\left(\mathbf{m V} @ \mathbf{m A ~} \mathrm{cm}^{-2}\right)\end{array}$ & $\begin{array}{l}\text { Tafel slope } \\
\left(\mathrm{mV} \text { dec }^{-1}\right)\end{array}$ & References \\
\hline 1 & $\mathrm{Rh}_{2} \mathrm{P}$ Nanocubes & $0.5 \mathrm{M} \mathrm{H}_{2} \mathrm{SO}_{4}$ & $5.4 @ 5$ & 29.9 & {$[1]$} \\
\hline 2 & $\mathrm{Rh}_{2} \mathrm{P}$ & $0.5 \mathrm{M} \mathrm{H}_{2} \mathrm{SO}_{4}$ & 14@10 & 31.7 & {$[2]$} \\
\hline 3 & Nanoparticles & $0.5 \mathrm{M} \mathrm{H}_{2} \mathrm{SO}_{4}$ & 38@10 & 38 & {$[3]$} \\
\hline 4 & $\mathrm{RuP}_{2} \mathrm{NPs} @ \mathrm{NPC}$ & $0.5 \mathrm{M} \mathrm{H}_{2} \mathrm{SO}_{4}$ & 13.10@10 & 30 & {$[4]$} \\
\hline 5 & $\mathrm{RuNPS} @ \mathrm{C}_{2} \mathrm{~N}$ & $0.1 \mathrm{M} \mathrm{HClO}_{4}$ & 15.8@10 & 29.9 & {$[5]$} \\
\hline 6 & $\mathrm{w}-\mathrm{Rh}_{2} \mathrm{P} \mathrm{NS} / \mathrm{C}$ & $0.5 \mathrm{M} \mathrm{H}_{2} \mathrm{SO}_{4}$ & 5.4@10 & - & {$[6]$} \\
\hline 7 & $\mathrm{Rh}_{2} \mathrm{P} / \mathrm{C}$ & $0.5 \mathrm{M} \mathrm{H}_{2} \mathrm{SO}_{4}$ & $\underline{2.0} @ 10$ & 10 & Present work \\
\hline & Rh-GO & & $\begin{array}{c}\text { (Lowest } \\
\text { overpotential) }\end{array}$ & & \\
\hline
\end{tabular}

Table S2: Evaluation summary of Oxygen Evolution Reaction belongs to state-of-the-art electrocatalysts reported in kinds of literature survey and present work.

\begin{tabular}{|c|c|c|c|c|c|}
\hline $\begin{array}{l}\text { Sr. } \\
\text { No. }\end{array}$ & Catalysts & Electrolytes & $\begin{array}{c}\text { Overpotential } \\
\text { a j(V@mA.cm-2) }\end{array}$ & $\begin{array}{l}\text { Tafel slope } \\
\left(\mathrm{mV} \text { dec }^{-1}\right)\end{array}$ & References \\
\hline 1 & $\mathrm{Rh}_{2} \mathrm{P} / \mathrm{C}$ & $1.0 \mathrm{M} \mathrm{H}_{2} \mathrm{SO}_{4}$ & $0.52 @ 5$ & - & [6] \\
\hline 2 & $\mathrm{CuNi}-\mathrm{C}$ & $1 \mathrm{M} \mathrm{KOH}$ & 0.40@10 & 83 & [7] \\
\hline 3 & Rh/SWNTs & $1 \mathrm{M} \mathrm{KOH}$ & 0.26@10 & 89 & [8] \\
\hline 4 & $\mathrm{G}-\mathrm{CO}_{3} \mathrm{O}_{4}$ & $1 \mathrm{M} \mathrm{KOH}$ & 0.31@10 & 56 & [9] \\
\hline 5 & $\mathrm{Co}-\mathrm{S}_{58} \mathrm{P}_{42}$ & $1 \mathrm{M} \mathrm{KOH}$ & 0.26@10 & 48 & {$[10]$} \\
\hline 6 & CoOx@CN & $1 \mathrm{M} \mathrm{KOH}$ & 0.26@10 & - & [11] \\
\hline 7 & Rh-GO & 0.5 М КОН & $0.23 @ 10$ & 27 & Present work \\
\hline
\end{tabular}




\section{Reference:}

(1) Duan H.; Li D.; Tang Y.; He Y.; Ji S.; Wang R.; Lv H.; Lopes P. P.; Paulikas A. P.; Li H. High-

Performance $\mathrm{Rh}_{2} \mathrm{P}$ Electrocatalyst for Efficient Water Splitting J. Am. Chem. Soc. 2017, 139, 54945502.

(2) Yang F.; Zhao Y.; Du Y.; Chen Y.; Cheng G.; Chen S.; Luo W. Monodisperse Rh ${ }_{2}$ P-Based Electrocatalyst for Highly Efficient and pH-Universal Hydrogen Evolution Reaction. Adv. Energy Mater. 2018, 8, 1703489.

(3) Pu Z.; Amiinu I. S.; Kou Z.; Li W.; Mu S. RuP 2 -Based Catalysts with Platinum-like Activity and Higher Durability for the Hydrogen Evolution Reaction at All pH Values. Angew. Chem. Int. Ed. 2017, $56,11559-11564$.

(4) Mahmood J.; Li F.; Jung S. M.; Okyay M. S.; Ahmad I.; Kim S. J.; Park N.; Jeong H. Y.; Baek J. B. An Efficient and pH-universal Ruthenium-Based Catalyst For The Hydrogen Evolution Reaction. Nat. Nanotech. 2017, 12, 441.

(5) Wang K.; Bolong H.; Lin F.; Lv F.; Luo M.; Zhou P.; Liu Q.; Zhang W.; Yang C.; Tang Y.; Yang Y.; Wang W.; Wang H.; Guo S. Wrinkled $\mathrm{Rh}_{2} \mathrm{P}$ Nanosheets as Superior $\mathrm{pH}-$ Universal Electrocatalysts for Hydrogen Evolution Catalysis, Adv. Energy Mater. 2018, 1801891.

(6) Duan H.; Li D.; Tang Y.; He Y.; Ji S.; Wang R.; Lv H.; Lopes P. P.; Paulikas A. P.; Li H.; Mao S. X.; Wang C.; Markovic N. M.; Li J.; Stamenkovic V. R.; Li Y.; High-Performance Rh ${ }_{2}$ P Electrocatalyst for Efficient Water Splitting. J. Am. Chem. Soc. 2017, 139, 5494-5502.

(7) Xie Z.; Wang Y.; Metal-Organic Framework-Derived CoNi-Embedded Carbon Nanocages as Efficient Electrocatalysts For Oxygen Evolution Reaction, Ionics, 2018, 24, 1773-1780.

(8) Zhang W.; Zhang X.; L.; Chen, Dai J.; Ding Y.; Ji L.; Zhao J.; Yan M.; Yang F.; Chang C.; Guo S.; Single-Walled Carbon Nanotube Induced Optimized Electron Polarization of Rhodium Nanocrystals To Develop an Interface Catalyst for Highly Efficient Electrocatalysis, ACS Catal. 2018, 8, 8092-8099. 
(9) Zhao Y.; Chen S.; Sun B.; Su D.; Huang X.; Liu H.; Yan Y.; Sun K.; Wang G.; Graphene-Co3O4 Nanocomposite As Electrocatalyst With High Performance For Oxygen Evolution Reaction Sci. Rep, 5 : 7629 | DOI: 10.1038/srep07629.

(10) Dai Z.; Geng H.; Wang J.; Luo Y.; Li B.; Zong Y.; Yang J.; Guo Y.; Zheng Y.; Wang X.; Yan Q.; Hexagonal-Phase Cobalt Monophosphosulfide for Highly Efficient Overall Water Splitting, ACS Nano $2017,11,11031-11040$

(11) Jin H.; Wang J.; Su D.; Wei Z.; Pang Z.; Wang Y.; In situ Cobalt-Cobalt Oxide/N-Doped Carbon Hybrids As Superior Bifunctional Electrocatalysts for Hydrogen and Oxygen Evolution, J. Am. Chem. Soc. $2015,137,2688-2694$. 